\title{
An Ambiguous Growth in Floor of the Mouth
}

\author{
Sukhjinder Kaur Khosa, Chaitanya D Nayak, Sonal Vahanwala, Sandeep S Pagare
}

\section{ABSTRACT}

Pathologic growths of the oral soft tissues are fairly common and include a diverse group of reactive and neoplastic conditions. Enlargement of or in relation to salivary glands have many causes, chief among them being inflammatory, neoplastic or physiological alterations. Nonspecific enlargement without any detectable cause has been classified as idiopathic and has been rarely reported. We describe a case of a 23-year-old female patient with a growth in floor of the mouth that was treated with conservative surgical excision. The histopathological features and differential diagnosis of this rare lesion are described and discussed. The awareness of this lesion is important to highlight the variations in the hyperplasias found in oral cavity for avoiding diagnosis and treatment pitfalls.

Keywords: Oncocytes, Salivary gland, Oncocytomas, Hyperplasia, Floor of the mouth, Cellular proliferation.

How to cite this article: Khosa SK, Nayak CD, Vahanwala S, Pagare SS. An Ambiguous Growth in Floor of the Mouth. Int J Head and Neck Surg 2013;4(1):51-54.

\section{Source of support: Nil}

\section{Conflict of interest: None}

\section{INTRODUCTION}

Pathologies associated with major and minor salivary glands are commonly reported in literature. Physiologic enlargements of the salivary glands are secondary to hormonal changes or age-related as in oncocytic hyperplasia. Nonspecific enlargements SANS physiological or pathological stimuli are rare and hitherto unreported. Salivary gland tumors constitute a heterogeneous group of lesions of a great morphological variation. Although uncommon, they are not so rare.
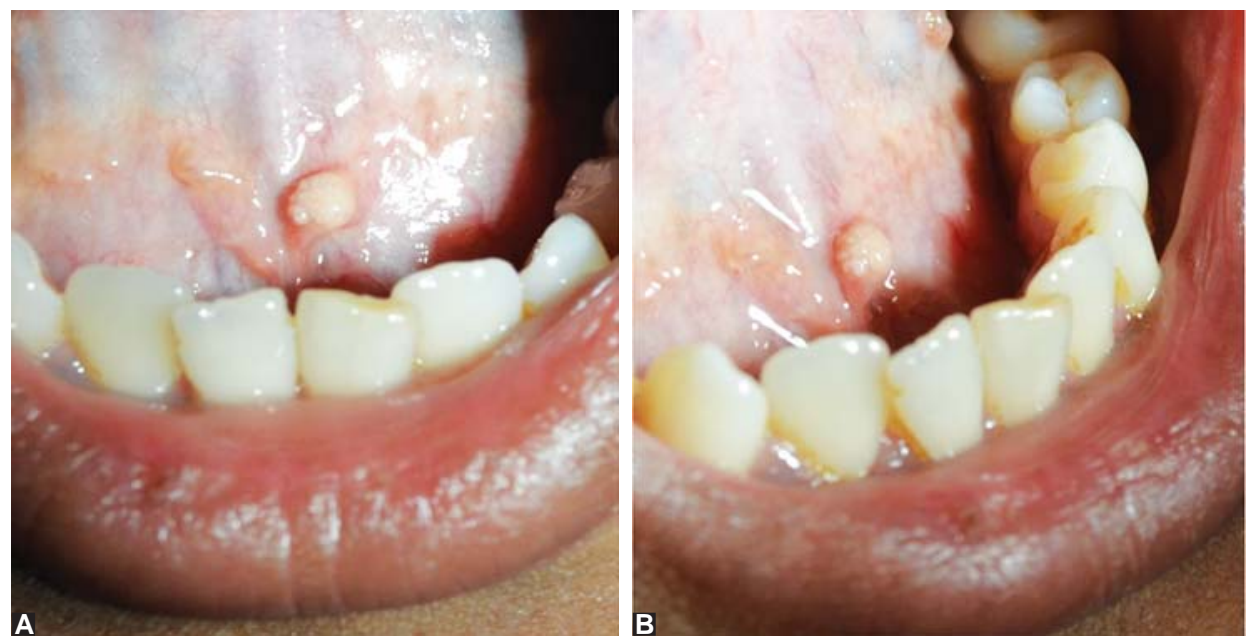

Figs 1A and B: (A) Nodular, pedunculated growth seen at the opening of the left Wharton's duct, (B) with pebbled surface and well-defined margins
Oncocytic neoplasms result from metabolically altered cells that accumulate abundant mitochondria within their cytoplasm by oncocytic metaplasia. These neoplasms rarely affect the major salivary glands, accounting less than $1 \%$ of all salivary gland tumors. They are thought to be the result of degeneration of salivary gland parenchyma or due to metaplastic process seen in hyperplasia of parenchyma. ${ }^{1}$ Therefore, oncocytes represent a special class of transformed epithelial cells that arise principally from glandular and secretory epithelia, and they are characterized by their large size and abundant, finely granular eosinophilic cytoplasm which is rich in mitochondrias. ${ }^{2}$

\section{CASE REPORT}

A 23-year-old female reported to the Department of Oral Medicine and Radiology with a complaint of an asymptomatic growth in the floor of the mouth. The growth was painless and was noticed by the patient for the past 1.5 year. The patient had no significant medical or drug history and no deleterious habits. Patient revealed that the growth started as small mass which gradually attained the present size and remained same from past 10 months.

Oral examination revealed a nodular, pedunculated growth of approximately $1 \mathrm{~cm}$ in diameter, freely mobile, nonulcerated and arising from the opening of the left Wharton's duct.

Clinically, growth was yellowish-white in color, with rough surface texture and pebbled surface with well-defined margins (Figs 1A and B). On palpation, the growth was 
nontender with soft to firm consistency. No localized lymphadenopathy was observed.

On the basis of clinical findings, differential diagnosis of salivary gland sialolith, verruca vulgaris, oral papilloma and condyloma acuminatum was given. Intraoral and panoramic radiographs revealed no obvious involvement of the mandible. Also, there was no evidence of calcifications of the salivary gland, thus, ruling out salivary gland sialolith.

The pebbled surface of the growth simulated the appearances seen with verruca vulgaris, oral papilloma and condyloma acuminatum, but the characteristic feature, of these being symmetric lesions having uniform pattern, can be used to distinguish it from the current case. The growth was excised in toto under local anesthesia taking care to avoid damage to the submandibular duct.

Histopathological examination (Figs 2A and B) of the excised mass showed sheets of oncocytes within the connective tissue stroma. These cells were large polyhedral in shape with abundant granular eosinophilic cytoplasm and dilated excretory duct was also evident. A histopathological diagnosis of oncocytic hyperplasia was returned.

The patient has been under observation and there are no signs of recurrence (Fig. 3).

\section{DISCUSSION}

Oncocytes were first described as eosinophilic, granular, swollen cells by Schaeffer in 1897, but later named and more fully characterized by Hamperl in $1931{ }^{3}$ Oncocytic lesions were described nearly a century ago by Schaffer when he described 'granular swollen cells' in the ductal and acinar elements of salivary glands. ${ }^{4}$ Hamperl is considered to be the 'Father of Oncocytes', originally referred to as 'oncocytes'. He chose this word because of the Greek root word (onkousthai), which means 'increase in bulk', swollen, enlarged or tumor. ${ }^{2,5}$ Hamperl described oncocytes in many organs including parotid, submaxillary, sublingual, and minor salivary glands, the thyroid, parathyroid, pituitary gland, adrenal gland, gallbladder, uterus, testicle, fallopian tube, pancreas, liver, stomach, kidney, lung, pharynx, trachea and esophagus. More recent publications reported oncocytes in the ovary, lacrimal caruncle, breast and thymus. ${ }^{5}$ He considered oncocytes to differ from other epithelial cells by their excessive proliferation of mitochondria. Because of their size and characteristic abundant acidophilic cytoplasm and altered DNA, oncocytic cells may be classified as somatic mutants. ${ }^{3}$ It is generally accepted that for a tumor to be called an oncocytoma, it must be comprised exclusively of oncocytes. There has been an active discussion in the literature as to whether oncocytomas are nodular hyperplasias or neoplasms. ${ }^{5}$

It has been demonstrated that oncocytic foci are rare before age 50 , and are almost universal after age 70 . Their number in the normal salivary gland increases with age. ${ }^{3}$ Oncocytes, originally thought to arise from a degenerative or senescent process, especially because oncocytes can be observed in otherwise normal specimens from aging patients, are now thought have been once thought to arise from a degenerative process are now thought to result from redifferentiation of epithelial cells which develop an increased but unbalanced metabolism due to an acquired enzymatic defect in the mitochondrial oxidative process trying to increase the output of high energy phosphate., ${ }^{2,5,6}$

Because mitochondria are somewhat independent organelles, another theory proposes that oncocytomas may
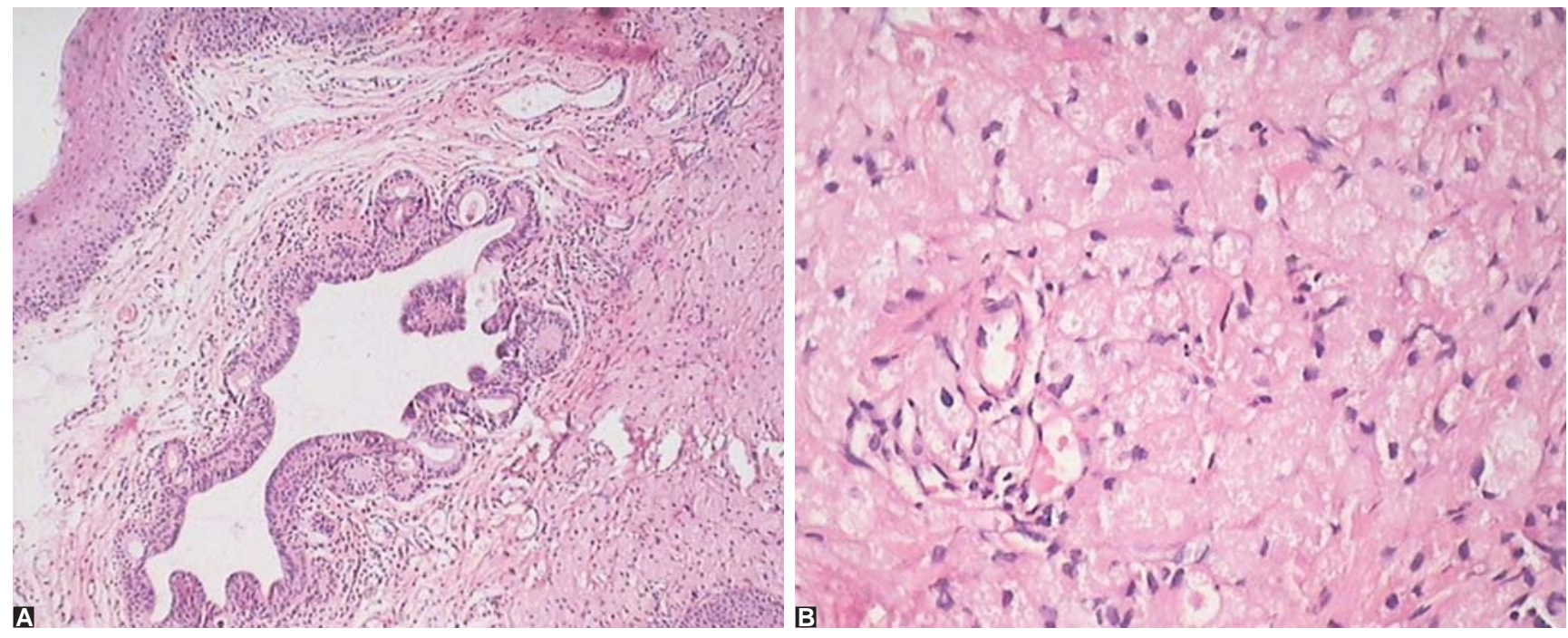

Figs 2A and B: (A) Ductal surface epithelium with connective tissue stroma and oncocytic proliferation, and (B) abundant granular eosinophilic cytoplasm 


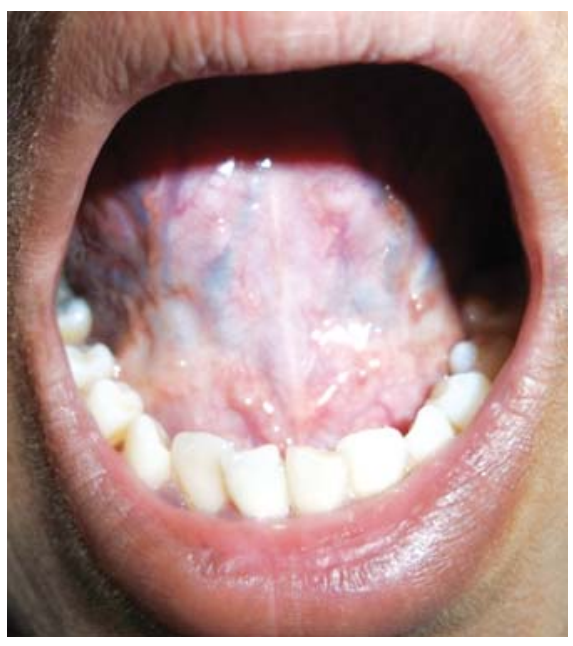

Fig. 3: Follow-up after one year

represent a neoplasm of subcellular organelles. Although an increase in mitochondria has been associated with increased activity, there has been no documented specific functionality or secretion for salivary gland oncocytomas. ${ }^{7}$

The World Health Organization (WHO) classification of salivary gland neoplasms recognizes three oncocytic entities: Oncocytosis, oncocytoma and oncocytic carcinoma. Oncocytosis is considered to be a hyperplastic change and may present with generalized enlargement of the glands whereas oncocytoma and oncocytic carcinoma represent neoplastic processes. ${ }^{8}$

Precisely, oncocytic hyperplasia (oncocytosis) is more frequent with age with a peak frequency of $14.7 \%$ between the ages of 61 and 70 years. $^{9}$

Oncocytic changes of secretory epithelia are thought to be metaplastic, a protective phenomenon against adverse change. Hamperl considered oncocytes to be burnt out cells as they lost their original specialization and increased in number with age. ${ }^{10}$ Bonikos DS et al suggested that oncocytic change may be the result of compensatory mitochondrial hyperplasia in normal cells caused by mitochondrial damage or the exhaustion of one or more mitochondrial enzymes. Linnane et al advocated that aging caused the accumulation of mitochondrial DNA errors leading to 'mitochondrial respiratory failure' and multisystem degeneration. ${ }^{11}$ Sunmunn et al stated that oncocytic change could be a regressive alteration of previously hypertrophic or hyperplastic ductal epithelium with the appearance of mitochondriopathy. ${ }^{12}$

The distinction between an oncocytic hyperplasia and an oncocytoma can be difficult histopathologically, especially in glands with multiple nodules. Oncocytoma can be distinguished from oncocytosis because it is well defined, solitary and commonly encapsulated. In contrast, oncocytosis is multifocal and does not disrupt nodular architecture of the glandular parenchyma. The presence of focal, ductal and acinar oncocytic metaplasia in other parts of the gland, in addition to presence of normal acini at the periphery of the large nodules in nodular oncocytic hyperplasia may be useful in distinguishing this lesion from true oncocytoma. 8,13,14 Criteria for the diagnosis of malignancy in salivary oncocytic tumors include: Distant metastasis, local lymph node metastasis, perineural, vascular or lymphatic invasion, frequent mitoses and cellular pleomorphism with extensive invasion and destruction of adjacent structures. $^{3}$

\section{CONCLUSION}

Oncocytic hyperplasia is considered to be one of the categories considered in proliferation of oncocytes, besides, oncocytoma and oncocytic carcinoma. The unusual occurrence of oncocytic hyperplasia in relation to submandibular gland in the second decade of life makes the present case, a rare variety. Although this lesion is very rare in occurrence, it should be considered in the differential diagnosis of atypical growths of the oral cavity. Complete surgical excision is the adequate therapy required, with no recurrence rate.

\section{REFERENCES}

1. Efthmia V, Apostolos T, Konstantinos D, George K, Ioannis. Parotid gland oncocytoma: A case report. Cases J 2009;2:6423.

2. Stump DJ. Onkocytic adenoma of the salivary gland. Arch Pathol 1949;48:287-96.

3. Gray SR, Cornog JL Jr, Seo IS. Oncocytic neoplasms of salivary glands: A report of 15 cases including two malignant oncocytomas. Cancer 1976;38(3):1306-17.

4. Schaffer J. Beitrage zur Histologie menschlicher Organe. IV. Zunge. V. Mundhohle-Schlundkopf. VI. Oesophagus. VII. Cardia. Sitzungsber D Kais Akad D Wissensch Math Naturwiss Classe Wien 1897;106(Part 3):353-455.

5. Lester DT, Bruce MW, Gary LE. Oncocytomas of the submandibular gland. A series of 22 cases and a review of the literature. Cancer 1996;78(11):2281-87.

6. Smith RA, Ord MJ. Mitochondrial form and function relationships in vivo: Their potential in toxicology and pathology. Int Rev Cytol 1983;83:63-134.

7. Cotton DWK. Oncocytomas. Histopathology 1990;16:507-09.

8. Hanna EY, Suen JY. Neoplasms of the salivary glands. In: Cummings C, Freidrickson J, Harker L, et al. (Eds). Otolaryngology-head and neck surgery (3rd ed). St Louis: Mosby 1998; 1256.

9. Cawson RA, Gleeson MJ, Eveson JW. Development anomalies, cysts and infiltrations in the pathology and surgery of the salivary glands (1st ed). Chapter 3. Oxford 1997;1-9.

10. Hamperl H. Benign and malignant oncocytoma. Cancer 1962;15:1019-27.

11. Bonikos DS, Bensch KG, Watt T, et al. Pulmonary oncocytes in prolonged hyperoxia. Exp Mol Pathol 1977;26(1):92-102.

12. Sakthi SP, Frank C, Alfredo A. Oncocytoma of the parotid gland and its mimickers: A comprehensive review. North Am J Med Sci 2010;3(4):171. 
13. Palmer TJ, Gleeson MJ, Eveson JW, Cawson RA. Oncocytic adenomas and oncocytic hyperplasia of salivary glands: A clinicopatholgical study of 26 cases. Histopathology 1990;16(5): 487-93.

14. Alberty J, August C, Stoll W. Oncocytic neoplasms of the parotid gland. Differential diagnosis, clinical course and review of literature. HNO 2001;49(2):109-17.

\section{ABOUT THE AUTHORS}

\section{Sukhjinder Kaur Khosa (Corresponding Author)}

Postgraduate Student, Department of Oral Medicine and Radiology Dr DY Patil Dental College and Hospital, Nerul (E), Navi Mumbai Maharashtra, India, e-mail: sukhjinderkaurkhosa@gmail.com

\section{Chaitanya D Nayak}

Professor, Department of Oral Medicine and Radiology, Dr DY Patil Dental College and Hospital, Navi Mumbai, Maharashtra, India

\section{Sonal Vahanwala}

Professor, Department of Oral Medicine and Radiology, Dr DY Patil Dental College and Hospital, Navi Mumbai, Maharashtra, India

\section{Sandeep S Pagare}

Professor, Department of Oral Medicine and Radiology, Dr DY Patil Dental College and Hospital, Navi Mumbai, Maharashtra, India 